\title{
La circulation de l'eau à travers la plante
}

\author{
Water movement through the plant
}

\author{
André Berger \\ Département de Physiologie Ecologique, CEPE, \\ CNRS, BP 5051, 34033 Montpellier Cédex
}

\section{Introduction}

Un continuel échange de matière et d'énergie a lieu entre la végétation et l'atmosphère, échange dont l'intensité dépend autant du climat que de l'état des plantes. Une partie de l'énergie reçue est irréversiblement fixée par la photosynthèse sous forme chimique. Si elle est négligeable en quantité, quand on écrit le bilan énergétique d'une masse de végétation, elle est par contre importante du point de vue production végétale. La plus grande partie de l'énergie absorbée par la végétation élève la température de celle-ci et est ensuite perdue sous forme de chaleur sensible et surtout de chaleur latente qui peut représenter entre 25 et $80 \%$ de l'énergie totale absorbée. Dans une végétation suffisamment dense, le flux de chaleur latente induit un flux d'eau qui transite, essentiellement à travers la plante, entre le sol et l'atmosphère. Ce flux liquide, qui sera seul envisagé dans cet exposé, transporte à travers la plante un certain nombre d'ions, absorbés dans le sol, contribuant ainsi à l'alimentation du végétal. Cette solution constitue ce que l'on appelle la sève brute circulant des racines vers les feuilles.

Des feuilles vers les racines ou vers d'autres organes s'établit un flux liquide transportant les substances organiques élaborées dans les feuilles (sève élaborée) empruntant des chemins parallèles à ceux de la sève brute (voir J.L. Bonnemain, même numéro).

Le volume d'eau qui transite dans la plante peut atteindre plusieurs litres d'eau par jour par $\mathrm{m}^{2}$ de sol couvert. Ce flux est plusieurs dizaines de fois plus important que le stock d'eau dans le végétal et notamment que la part de stock qui peut être mobilisée pour contribuer à la transpiration générale. L'idée première a donc été d'utiliser un modèle à flux conservatif pour traiter la circulation de l'eau à travers la plante. Cet exposé sera essentiellement consacré à la description de ce modèle général et à l'analyse de ses limites.
Cependant, le stock d'eau de la plante variant dans la journée, ses variations provoquent des contraintes hydriques qui modifient la physiologie du végétal. Le problème des variations de stock dans les différents compartiments de la plante est abordé par P. Cruiziat (même numéro).

Comme les évolutions journalières et saisonnières de l'état hydrique sont importantes dans le fonctionnement de la plante, il était du plus grand intérêt pour le physiologiste et l'agronome de connaître et éventuellement prévoir ces variations. L'analyse des facteurs agissant sur l'état hydrique des feuilles a donc été entreprise d'abord sur la base d'un modèle très général.

\section{Modèle général}

L'ensemble des études expérimentales effectuées ces dernières décennies montre que l'état hydrique des feuilles dépend, à la fois des conditions hydriques dans le sol, dans l'atmosphère, et de caractéristiques structurales et fonctionnelles du végétal. De nombreux auteurs ont utilisé pour rendre compte de ces observations un modèle linéaire simplifié, représenté par la relation :

$$
\psi_{p l}=\psi_{s}-\mathrm{FR}
$$

relation dans laquelle $\psi_{p l}$ et $\psi_{s}$ sont respectivement le potentiel hydrique dans la plante et dans le sol, $F$ le flux liquide entre le sol et la plante et $R$ la résistance à la circulation de l'eau entre ces deux zones ${ }^{(1)}$. En toute rigueur, il faudrait rajouter au terme $\psi_{s}$, le terme $\psi_{g}$

(1) Le potentiel hydrique caractérise l'énergie de liaison de l'eau avec le substrat et s'exprime en erg $g^{-1}$ et plus souvent en bars. 
qui représente la composante liée à la gravité et souvent omis sur les végétaux de faible taille.

Un certain nombre de résultats expérimentaux conduisent à une relation entre $\psi_{p l}$ et $F$, qui peut être considérée comme linéaire (Fig. 1 , Fig. 2 et Fig. 3A), montrant une chute de potentiel liée à l'augmentation de transpiration (Neumann et al., 1974).

Certains auteurs ont cependant, observé un $\psi$ constant quel que soit le flux traversant la plante (Fig. 3D) (Camacho et al., 1974 et Trouchton et al., 1974). D'autres résultats montrent une relation complexe dans laquelle le potentiel chute rapidement dans la gamme des faibles flux puis se stabilise (Tinklin et Weatherley, 1966) (Fig. 3C). Nous avons également montré sur tournesol (Berger, 1971) et sur pin pignon (Berger et Ibrahim, résultats non publiés) une relation linéaire dans une large gamme de flux puis une tendance à la stabilisation du potentiel (Fig. 3B).

Enfin, il faut signaler que, dans de nombreux cas, un effet d'hysteresis se manifeste, tel que pour des flux égaux on observe des potentiels différents suivant que l'on se trouve en phase d'augmentation du flux (matin) ou en phase de diminution (fin de journée).

Avant d'entreprendre une analyse critique de la signification de ces différentes relations, il nous faut décrire les trajets suivis par l'eau dans la plante, les principales structures de transfert et les forces qui sont à l'origine de ces transferts.

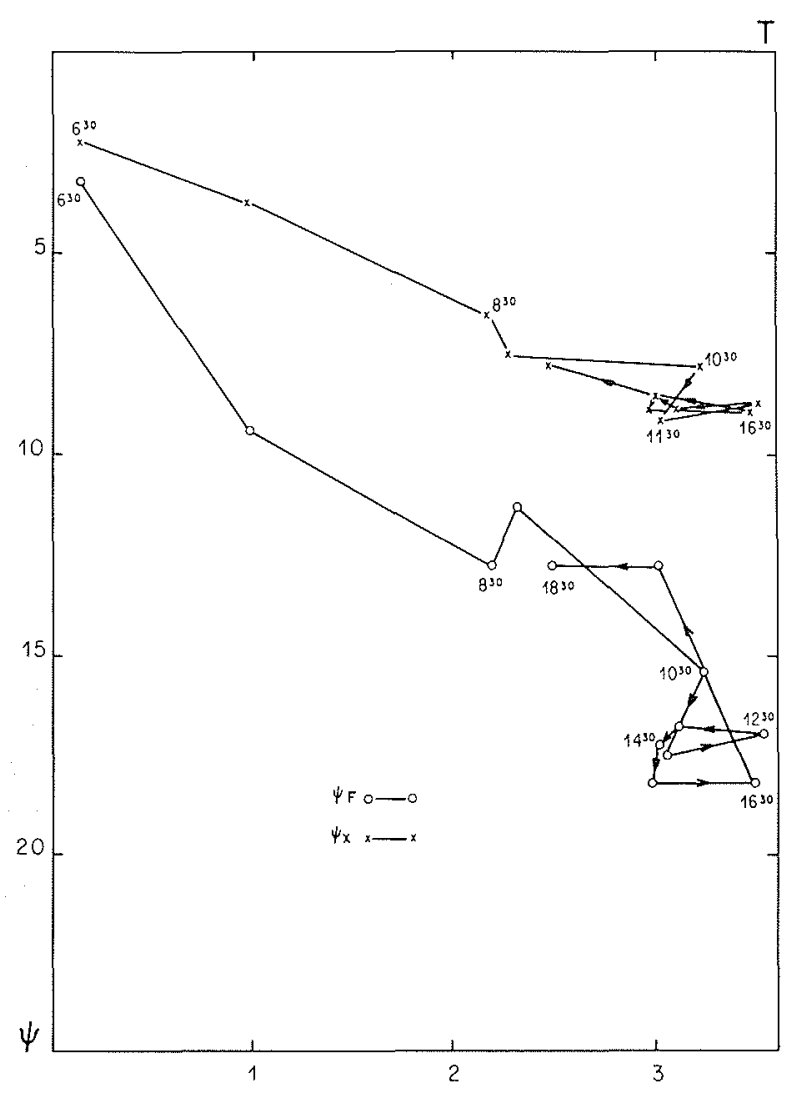

Figure 1 - Relation entre le potentiel hydrique, en bars, des feuilles $\left(\psi_{f}\right)$ ou de la vascularisation $\left(\psi_{x}\right)$, et la transpiration $(T)$ exprimée en $\mathrm{g}$ d'eau par minute et par $\mathrm{m}^{2}$ de surface foliaire. Pour la signification respective de $\psi_{F}$ et $\psi_{x}$ voir figure 2 . En face des points est portée l'heure moyenne de mesure.

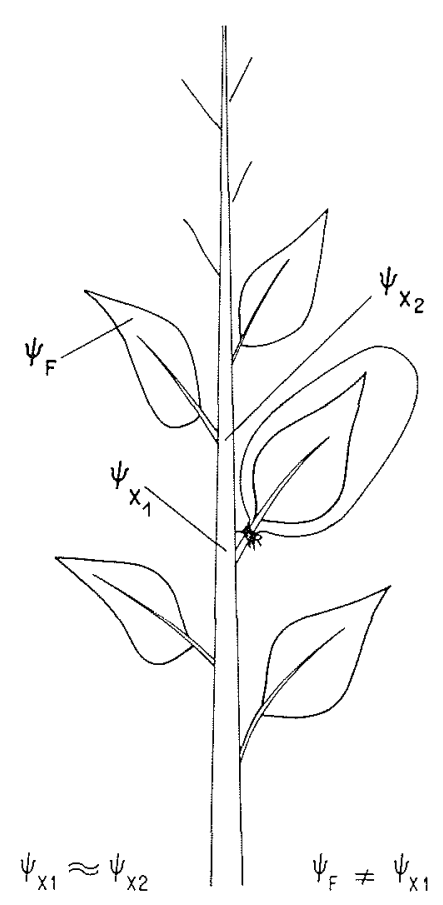

Figure 2 - Chez le pommier l'existence de rameaux longs, comportant au plein développement de 15 à 30 feuilles, permet d'étudier la répartition des potentiels le long du rameau. Il est possible notamment de mesurer le potentiel des feuilles dont la transpiration a été bloquée pendant quelques dizaines de minutes par une enveloppe étanche. Ce potentiel s'équilibre alors avec celui existant au point d'insertion de la vascularisation foliaire sur la vascularisation principale du rameau. La valeur obtenue est appelée $\psi_{x}$. L'expérience montre que les valeurs de $\psi_{x}$ sur deux feuilles successives sont très voisines (différence $<2$ bars), par contre la différence $\psi_{F}-\psi_{x}$ sur deux feuilles successives peut atteindre, dans les mêmes conditions, 5 à 8 bars. $\psi_{F}$ étant le potentiel de la feuille dont la transpiration n'est pas bloquée.

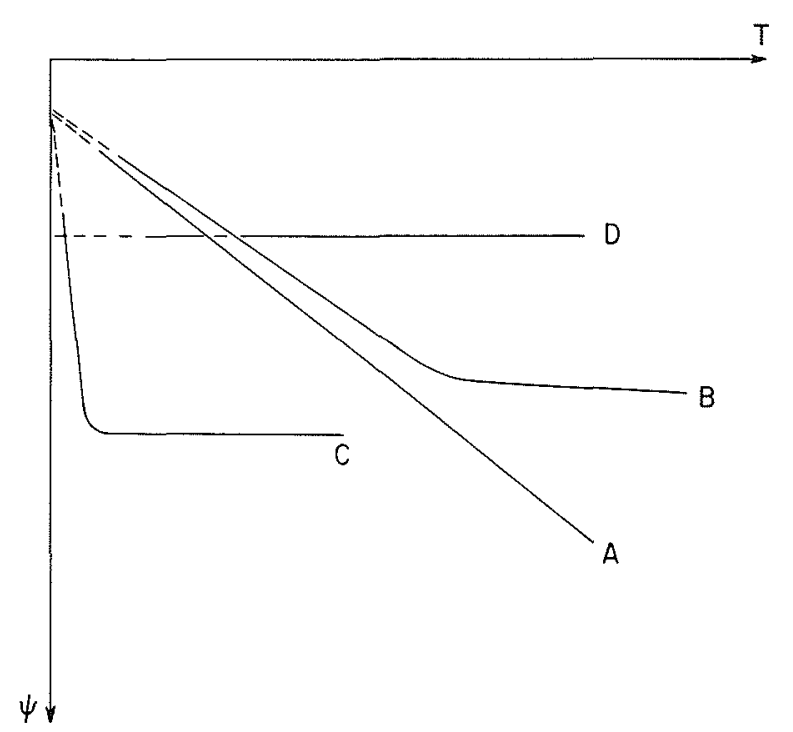

Figure 3 - Principaux types de relation $\psi=f(T)$ obtenus sur différentes espèces et dans différentes conditions (voir références bibliographiques dans le texte). 


\section{Les structures de transfert}

Entre le sol et la surface d'évaporation située dans les feuilles, l'eau traverse un premier massif cellulaire au niveau de la racine, un système conducteur qui transporte l'eau de la racine vers les feuilles, et enfin un autre massif cellulaire dans les feuilles.

$\mathrm{Au}$ niveau cellulaire, deux voies possibles de circulation de l'eau se présentent. Soit l'eau traverse la cellule et doit donc franchir un certain nombre de barrières membranaires, soit elle contourne la cellule, en circulant dans la paroi cellulosique qui l'entoure. Dans ce dernier cas, le flux principal ne traverse pas la cellule et seuls des échanges mineurs ont lieu entre la cellule et flux privilégié pariétal. Dans l'état actuel de nos connaissances la seconde hypothèse semble devoir être retenue. Morizet détaille plus loin l'importance relative de ces deux voies possibles (même numéro).

\section{Pénétration à travers la racine}

Le massif cellulaire qui, dans la racine, sépare le sol du système conducteur de la sève brute est composé d'un certain nombre de couches cellulaires dont la structure pariétale constitue un continuum pouvant assurer presque intégralement la conduction de l'eau. On observe, en effet, un épiderme muni de poils absorbants qui assurent le contact racine-sol, puis une zone corticale sans particularité anatomique et enfin une zone circulaire entourant l'ensemble des vaisseaux conduc-

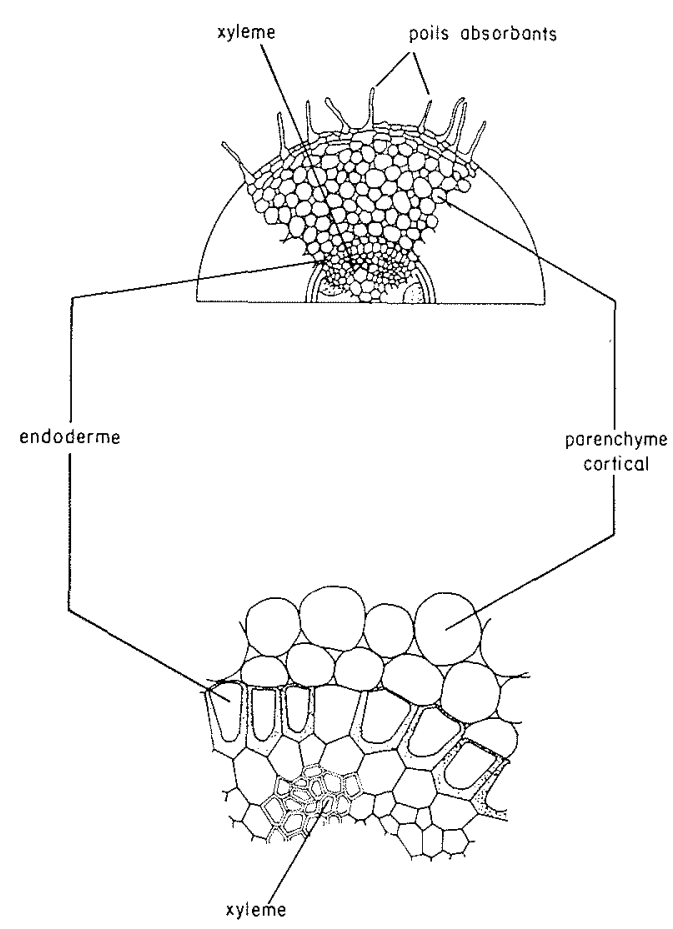

Figure 4 - Coupe d'une racine présentant les diverses zones franchies par l'eau depuis l'épiderme jusqu'aux vaisseaux de xylème qui conduisent la sève brute vers les parties aériennes. La zone endodermique est agrandie de façon à montrer la structure détaillée de l'endoderme.

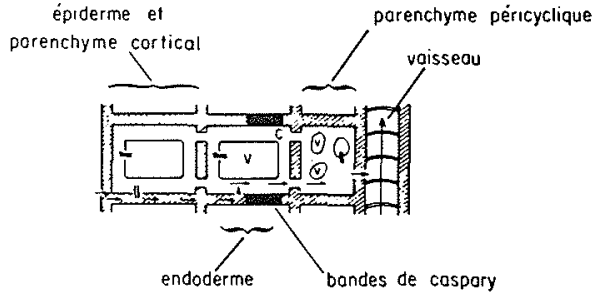

Figure 5 - Coupe schématique d'une racine sur laquelle est portée la principale voie de circulation de l'eau entre le sol et les vaisseaux.

teurs. Cette couche monocellulaire (endoderme) présente une modification telle des parois transversales (bandes de Caspary) que la voie pariétale de circulation de l'eau est interrompue et qu'à ce niveau une pénétration cellulaire est indispensable (Fig. 4 et 5).

Cette pénétration obligée, dans la couche cellulaire, est à l'origine d'une résistance importante à la circulation de l'eau à travers les racines, mais a cependant une signification adaptative certaine. Elle est la base du système de contrôle de pénétration de substances diverses, contenues dans la solution qui imprègne les parois.

\section{Circulation dans le xylème}

Le système conducteur de la sève brute des racines vers les feuilles est constitué de cellules vides, d'un diamètre de 10 à $500 \mu \mathrm{m}$ et de quelques centaines de $\mu \mathrm{m}$ à quelques millimètres de longueur. Cette zone offre à la circulation de l'eau une résistance relativement faible.

\section{Circulation dans la feuille}

Les nervures, parties terminales du système conducteur, amènent dans la feuille l'eau, qui doit traverser ensuite un massif cellulaire, non vascularisé, avant d'aboutir dans la zone d'évaporation. Ici la voie pariétale n'est interrompue par aucune structure de type endodermique (Fig. 6).

Du fait de leur composition anatomique particulière, ces trois structures de transfert offrent des résistances différentes à la circulation de l'eau, en phase liquide. Un ordre de grandeur des conductivités nous est donné par les chiffres suivants (Nobel, 1974). La chute de potentiel hydrique nécessaire pour assurer un flux de $0,1 \mathrm{~cm}^{3} \mathrm{~cm}^{-2} \mathrm{~s}^{-1}$ est de $2 \cdot 10^{-4}$ bar, pour un vaisseau conducteur de $1000 \mu \mathrm{m}$ de longueur et de $20 \mu \mathrm{m}$ de rayon, 30 bars pour la traversée d'une paroi $(10 \mu \mathrm{m}$ d'épaisseur) et $2.10^{4}$ bars pour la traversée d'une membrane telle que le plasmalemme.

Il faut signaler enfin une dernière structure de transfert, l'épiderme, qui intéresse la diffusion de la vapeur d'eau évaporée dans les méats intercellulaires de la feuille. L'épiderme est constitué d'une couche de cellules dopt la paroi externe, plus ou moins cutinisée, est relativement imperméable à la diffusion des gaz. Les échanges sont assurés par une population de pores, les 

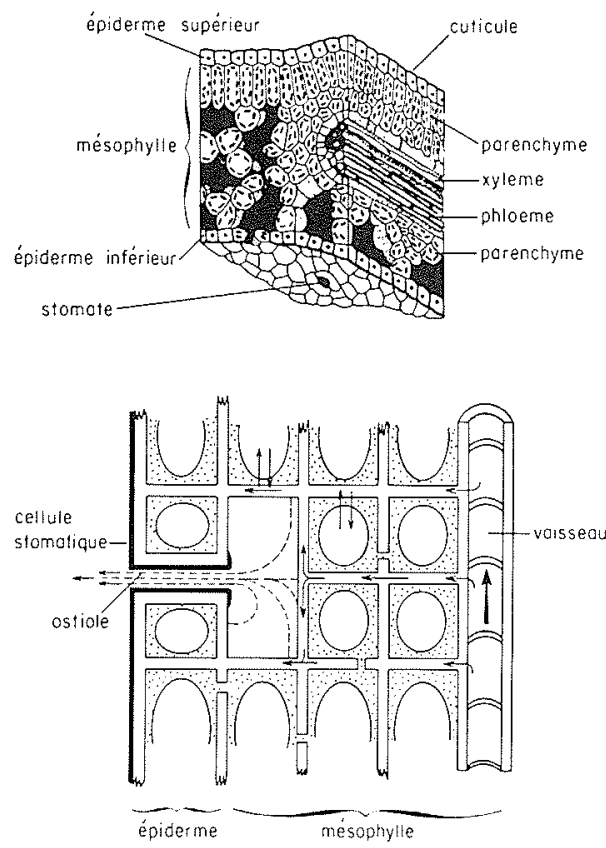

Figure 6 - Coupe d'une feuille au niveau de la partie terminale d'une nervure et schéma correspondant sur lequel sont portées les voies de circulation de l'eau entre les vaisseaux et l'atmosphère. En traits pleins la circulation en phase liquide, en tiretés la diffusion en phase gazeuse.

stomates, qui perforent cet épiderme et dont le diamètre varie en réponse à un certain nombre de facteurs externes à la plante (lumière, température, humidité de l'air) et internes à celle-ci (état hydrique, teneur en $\mathrm{CO}_{2}$ des méats foliaires, balance hormonale etc.).

\section{Les forces motrices}

La différence d'énergie de liaison de l'eau entre deux points d'un système est à l'origine de la force motrice qui fait circuler celle-ci. Nous avons déjà vu plus haut que la notion de potentiel hydrique est utilisée pour décrire cette énergie de liaison de l'eau et la différence de potentiel devient l'élément moteur de la circulation de l'eau. Le potentiel de l'eau libre, à la température et à la pression de l'expérience, étant pris comme potentiel de référence égal à 0 , les potentiels observés sont le plus souvent négatifs.

Un certain nombre de phénomène physico-chimiques tels qu'interactions osmotiques avec des substances dissoutes $(\pi)$, interactions capillaires avec des substances non dissoutes $(\tau)$, action de pression $(P)$ ou de tension $(T)$ externes au système, contribuent à modifier le potentiel hydrique.

La relation générale :

$$
\psi=\pi+\tau+P+T
$$

permet de relier les principales composantes du potentiel hydrique d'un système. Deux zones d'un système peuvent avoir un potentiel global égal, tout en ayant des composantes très différentes.
Ainsi dans un végétal deux grands territoires existent, milieu extracellulaire (vaisseaux et parois) et intracellulaire (cy toplasme, vacuoles) qui, même à l'équilibre de potentiel, ont des caractéristiques très différentes.

Dans le milieu extracellulaire les paramètres capillaires $(\tau)$ ou hydrostatiques $(T)$ sont les composantes essentielles et fortement négatives du système. $\pi$ pour sa part apporte une contribution assez faible, la sève brute étant relativement peu concentrée

$$
\begin{array}{ll}
\psi=-20 & \pi=-2 \\
& \tau=T=-18
\end{array}
$$

Dans le milieu intracellulaire, par contre, un tel potentiel global sera obtenu par la combinaison d'un potentiel osmotique vacuolaire très négatif et d'un potentiel hydrostatique positif assez élevé.

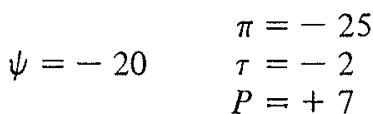

Il est évident que la différence de potentiel osmotique entre ces deux milieux ne peut se manifester que parce qu'ils sont séparés par une membrane à perméabilité sélective.

De nombreuses méthodes existent pour mesurer le potentiel global des tissus ou des organes mais aucune n'est totalement satisfaisante. Deux cependant ont actuellement la faveur des auteurs.

La première consiste à compenser, sur une tige feuillée, les tensions hydrostatique $(T)$ et capillaires $(\tau)$ par une pression externe $(P)$. Cette pression ramène à la surface de la section la colonne de sève brute qui, lors de la section, s'était brusquement retirée à l'intérieur de la vascularisation. Cette méthode donne une valeur très voisine de $\psi$ dans le milieu extracellulaire puisque la composante $\pi$ y est en général négligeable.

La deuxième méthode permet d'appréhender le potentiel moyen d'un tissu, quelle que soit la part relative des milieux extra et intra cellulaires. Elle consiste à mettre l'échantillon prélevé en équilibre de tension de vapeur d'eau avec l'atmosphère qui le surmonte. Lorsque cet équilibre est atteint, ce qui peut demander un temps de l'ordre de l'heure, le point de rosée est mesuré dans l'atmosphère. La durée de la phase de mise en équilibre assure que tout gradient de potentiel entre différentes parties de l'échantillon est effacé et que l'on obtient une valeur moyenne du potentiel.

\section{Lois de circulation}

La loi de base régissant la circulation de l'eau est de la forme (Dainty, 1969) :

$$
J_{w}=L_{p} \Delta \psi
$$

relation dans laquelle $J$ est le flux volumique d'eau en $\mathrm{cm}^{3} \mathrm{~cm}^{2} \mathrm{~s}^{-1}, \Delta \psi$ la différence de potentiel en bars et $L_{p}$ la conductivité hydraulique en $\mathrm{cm} \mathrm{s}^{-1} \mathrm{bar}^{-1}$.

Pour tenir compte de toutes les composantes du potentiel hydrique dans le végétal, une loi généralisée est applicable aux échanges entre la cellule et le milieu

$$
J_{w}=L_{p}\left(\Delta P-\Delta \pi_{\text {imp }}-\sigma \Delta \pi_{p}-\Delta \tau\right)
$$


relation dans laquelle $J_{w}$ et $L_{p}$ ont la même signification que précédemment, $\Delta P$ la différence de potentiel hydrostatique, $\Delta \pi_{\mathrm{imp}}$ la différence de potentiel osmotique due aux solutés non perméants, $\Delta \pi_{p}$ la différence de potentiel osmotique due aux solutés perméants, $\Delta \tau$ la différence de potentiel due aux interactions capillaires et $\sigma$ le coefficient de réflexion de la membrane exprimant le fait que les solutés responsables de $\Delta \pi_{p}$ n'exercent pas totalement la pression osmotique liée à leur concentration.

$L_{p}$ entre la cellule et le milieu externe est souvent mesurée par des expériences de déplasmolyse (Stadelman, 1966). Le résultat obtenu correspond en fait à la conductivité hydraulique d'un système constitué par la paroi, le plasmalemme, le protoplasme, et le tonoplaste en série. Dans des systèmes multi-cellulaires, tels qu'un appareil racinaire la signification de la mesure de $L_{p}$ est plus complexe.

\section{Relations entre structures de transfert et potentiel}

Le modèle linéaire proposé pour étudier les relations entre débits et potentiels suppose, implicitement, et ce point est en fait rarement envisagé, une stabilité fonctionnelle des structures de transfert quelles que soient les valeurs de potentiels. A-t-on actuellement des raisons de penser qu'il en est ainsi et sinon quelles sont les déformations que peuvent subir ces structures et quels sont les mécanismes physico-chimiques intervenant ?

\section{Milieu extracellulaire}

Ces voies de transferts, nous l'avons vu, sont essentiellement constituées par les capillaires des parois et surtout les vaisseaux du xylème. Lorsque la tension $(T)$ dans la tige s'élève, au cours de la journée, pour atteindre des valeurs élevées, des variations de diamètres sont observées. Ces variations pourraient s'interprêter en terme de variation de diamètres des vaisseaux conducteurs puisquils sont le siège des variations de tension. En fait, des travaux récents ont montré que la variation du diamètre de la tige est essentiellement due à une variation d'épaisseur de la zone cambium + liber (Fig. 7). Ainsi, la conductivité hydraulique du xylème ne semble pas devoir être directement affectée par les variations de tension hydrostatique qu'elle subit.

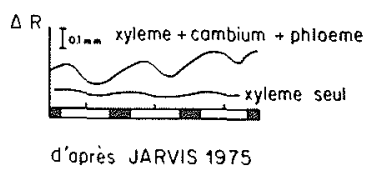

Figure 7 - Variations journalières du rayon de la tige intacte (xylème + cambium + phloème) et de la tige décortiquée (xylème) chez l'Epicéa. Sur l'axe des abcisses, en blanc la phase diume, en noir la phase nocturne.
Il ne faut cependant pas exclure l'existence de phénomènes de cavitation pouvant réduire la conductivité du système de telle façon que la circulation de l'eau, lors de réhumectation des tissus soit rendue plus difficile (voir P. Cruiziat, même numéro).

\section{Milieu intracellulaire}

La cellule végétale constitue un système déformable, qui peut être caractérisé par un certain nombre de relations hydrodynamiques.

La première n'est que la transposition au niveau cellulaire de la relation (2) :

$$
\psi_{\text {cell }}=\pi_{\text {cell }}+\tau_{\text {cell }}+P
$$

relation dans laquelle $\psi_{\text {cell }}$ représente le potentiel hydrique total de la cellule, $\pi_{\text {cell }}$ son potentiel osmotique, $\tau_{\text {cell }}$ son potentiel matriciel et $P$ son potentiel hydrostatique ou potentiel de turgescence.

Les variations de potentiel de turgescence correspondent aux variations de volume liées aux pertes et aux gains en eau. Les relations expérimentales entre $P$ et les variations de volume cellulaire, quoique relativement complexes, sont de la forme générale :

$$
P=k\left(\frac{V-V_{0}}{V_{0}}\right)
$$

avec $V_{0}$, le volume de la cellule lorsque le potentiel de turgescence est nul, $V$ le volume cellulaire et $k$ un paramètre qui représente le module d'élasticité de la paroi cellulaire. Des résultats récents semblent montrer que $k$ dépend fortement du potentiel de turgescence $(P)$.

Certains auteurs ont également envisagé l'hypothèse selon laquelle ces déformations pourraient induire des modifications du paramètre $L_{p}$ (Zimmerman et al., 1974) (voir relation (4)). La question reste encore controversée puisque Steudle et al. (1975) et Palta et al. (1976) observent une faible variation de $L_{p}$.

Analyse critique des différences entre le modèle simplifié et les diverses relations expérimentales

Le panorama de l'ensemble des résultats expérimentaux montre une grande diversité de réponse dans la relation Flux-Potentiel et donc une divergence pour certains résultats avec le modèle linéaire envisagé au début de l'exposé. Il semble y avoir deux types de causes de divergence; l'un tient à une assez grande variété des conditions expérimentales, l'autre à l'existence de phénomènes physico-chimiques, liés à la complexité du végétal, et non pris en compte dans le modèle simplifié.

\section{Conditions expérimentales et méthodes de mesure}

Un certain nombre de résultats ont été obtenus avec le végétal cultivé dans un sol, beaucoup d'autres corres- 
pondent à des cultures en milieu liquide. Dans ce dernier cas, est éliminé l'effet d'une résistance à la circulation de l'eau entre le sol et la racine. Cet effet peut devenir important lorsque sous l'action de la tension intravasculaire, les racines voient leur diamètre diminuer, jusqu'à $40 \%$ parfois, ce qui entraîne probablement une réduction du contact racine-sol (Huck et al., 1970). Certaines variations de résistance, observées au cours de la joumée, et le phénomène d'hystéresis correspondant, pourraient s'expliquer par ce mécanisme, dont l'impact réel n'est pas encore définitivement montré (Camacho et al., 1974).

L'ensemble des résultats, correspondant à des cultures en milieu liquide, presente des non linéarités qui doivent être expliquées à partir d'autres causes expérimentales ou fondamentales.

Les techniques de mesure utilisées posent un problème méthodologique, car elles peuvent être à l'origine de décalages qui déforment la relation $\psi=f$ (transpiration). Les mesures de transpiration sont la plupart du temps effectuées par pesées successives de pots contenant le végétal. Les valeurs obtenues correspondent à une moyenne sur quelques minutes, voire quelques dizaines de minutes. Par contre la mesure de potentiel donne la valeur de celui-ci à l'instant où l'échantillon végétal a été prélevé. Des prélèvements trop fréquents étant exclus, il est difficile d'avoir une moyenne correcte lorsque le végétal est soumis à un régime transpiratoire éloigné du régime permanent.

Ces réflexions ont amené à définir des conditions expérimentales susceptibles de réduire ces causes de divergence :

- étude en régime permanent et travail par paliers successifs de transpiration,

- mesure en continu du potentiel hydrique dans le milieu extracellulaire des feuilles.

Ceci a été réalisé sur tournesol, maïs et soja, par Neumann et al. (1973) qui observent dans tous les cas une très bonne relation linéaire.

\section{Phénomènes physico-chimiques non pris en compte dans le modèle simplifié}

En conditions de régime permanent Tinklin et al. (1966) ont cependant mis en évidence une variation rapide de $\psi$ pour les faibles flux, suivie d'une variation moins élevée pour les flux plus élevés. Cette observation corrobore celle de Brouwer (1953) et Kuiper (1963) qui montrent que lorsque le flux à travers une racine augmente, la résistance à celui-ci diminue.

Signalons cependant que la variation de résistance n'est mise en évidence que pour les faibles débits ; audelà d'un certain seuil la résistance reste stable. Ceci explique que, pour les débits observés dans les conditions naturelles, le phénomène passe inaperçu et puisse être négligé dans les études écophysiologiques ou bioclimatologiques. Il est, par contre, d'un grand intérêt d'étudier ce phénomène pour approfondir notre compréhension de la structure et du fonctionnement de l'appareil racinaire.
Pendant quelques années la seule explication disponible de cette non linéarité était l'existence du "phénomène Erbes". Dans ce phénomène, la non linéarité serait due au fait que lorsque le débit augmente, c'està-dire la différence de tension entre les deux faces de l'endoderme, le nombre de pores conducteurs au niveau des membranes augmente ; ceci évidemment jusqu'à ce que tous les pores disponibles soient fonctionnels.

Plus récemment, Dalton (1972) et Fiscus (1975) refusent d'accepter cette explication qui, effectivement, n'a pas eu de confirmation expérimentale. Ils envisagent dans leur modèle l'interaction des transports de solutés et d'eau au niveau de la racine et les effets d'entraînement.

Indépendament de ces interactions la résistance du système est de la forme :

$$
R=\frac{1}{L_{p}}
$$

Fiscus montre que si l'on envisage les échanges d'eau et de solutés, la résistance apparente dans le système devient :

$$
R_{a}=\frac{1}{L_{p}}+f\left(J_{s}^{*}, J_{v}\right)
$$

relation dans laquelle $J_{s}^{*}$ est le transfert actif de solutés, $J_{v}$ le flux total de matière,

Ce dernier modèle rend compte de la plupart des résultats obtenus concernant la circulation de l'eau et d'un certain nombre concernant les transports de solutés. Il demande, cependant, à être testé par une étude expérimentale qui envisagerait simultanément les flux d'eau et de substances ainsi que les potentiels et les concentrations.

\section{Conclusion}

Ainsi, le modèle linéaire, bien commode par ailleurs, n'est pas toujours applicable, pour un certain nombre de raisons qui tiennent à l'existence de :

- Conditions expérimentales qui introduisent des résistances variables supplémentaires (par exemple : interface racine-sol)

- Régime transitoire, très fréquent en conditions naturelles où le pouvoir évaporant de l'air varie énormément au cours de la journée

- Interactions importantes entre le flux des solutés et celui de l'eau.

Nombre de ces aspects sont détaillés dans les communications qui suivent.

\section{Bibliographie}

BERGER A. (1971) - Etude de la circulation de l'eau dans le système sol-plante. Thèse de Doctorat d'Etat, Université de Montpellier. 
BROUWER R. (1953) - Water absorption by the roots of Vicio foba at various transpiration strenght. II. Crucial relation between suction tension, resistance and uptake. Proc. K. Ned. Akad. Wet. Sci. Co. 56, 129-136.

CAMACHO-B. S.E., KAUFMANN M.R. and HALL A.E. (1964) Leaf water potential response to transpiration by citrus. Phy. siol: Plant, 31, 101-105.

DAINTY J. (1969) - The water relations of plants. In : Physiology of plant growth and development. M.B. Wilkins ed.

DALTON F. (1972) - A physical-mathematical model describing the simultaneous transport of water and solutes accross root membranes. Ph. D. thesis University of Wisconsin.

FISCUS E.L. (1975) - The interaction between osmotic and pressure induced water flow in plant roots. Plant Physiol. $55,917-922$

HUCK M.G., KLEEPPER B. and TAYLOR M.M. (1970) Diurnal variations in root diameter. Plant Physiol., 45, 529 . 530.

KUIPER P.J.C. (1963) - Some considerations on water transport accross living cell membranes: 59-68. In: J. Zelitch(ed) Stomato and water relations in plants. Bull. 664 , Conn. Agr. Exp. Sta., New-Haven, Conn.

NEUMAN H.H., THURTELL G.W. and STEVENSON K.R. (1973) - In situ measurements of leaf water potential and resistance to water flow in corn, soybean and sunflower at several transpiration rates. Can. J. Plant Sci., 54, 175-184.
NOBEL P.S. (1974) - Introduction to biophysical plant physiology. N.H. Freeman and Company, San Francisco. 488 p.

PALTA J. and STADELMANN E.J. (1976) - The effect of turgor pressure on water permeability of Allium cepa epidermis cell membranes. Plant Physiol., 57, Ann. Meeting Suppl.p. 79.

STADELMAN E.J. (1966) - Evaluation of turgidity, plasmolysis and deplasmolysis of plant cells. In: Methods in cell physio$\operatorname{logy}$ (ed. D.M. Prescott). Vol. Il, p. 143-216, New-York, London Academic Press.

STEUDLE E., LUTTGE U. and ZIMMERMANN U. (1975). Water relations of the epidermal bladder cells of the halo. phytic species Mesembryanthemum crystallinum. Direct measurements of hydrostatic pressure and hydraulic conductivity, Planta, 126, 229-246.

TINKLIN R, and WEATHERLEY P.E. (1966) - On the relationship between transpiration rate and leaf water potential. New Phytol., 65, 509-517.

THROUGHTON JM., CAMACHO-B S.E. and HALL A.E. (1974) - Transpiration rate, plant water status and resistance to water flow in Tidestromia oblongifolia. Carnegie Inst. Yearbook, 73, 830-835.

ZIMMERMANN U. and STEUDLE E. (1974) - Hydraulic conductivity and volumetric elastic modulus in giant algal cells: Pressure and volume dependence. In: Membrane transport in plants (eds: U. Zimmermann, J. Dainty), p. 64-71, Berlin-Heidelberg-New-York: Springer.

\section{Discussion}

Président : M.S. HENIN

Le Président remercie M. BERGER d'un exposé qui, en présentant clairement une série de modèles simples, devrait épargner beaucoup des discussions à venir.

A la demande de M. KOPP, M. BERGER définit comme suit le "potentiel" :

Le potentiel caractérise l'état énergétique de l'eau; c'est l'énergie qu'il faut fournir au système pour en extraire une cer taine quantité d'eau libre. Je prends comme analogie une éponge humide : il faut exercer une certaine pression pour extraire une certaine quantité d'eau. Le potentiel couramment exprimé en bars correspond à cette pression.

Le Président précise que le potentiel comporte trois composantes: le potentiel osmotique, le potentiel capillaire, le potentiel "hydrostatique".

M. CRUIZIAT observe que le potentiel hydrique est en réalité une énergie par unité de volume d'eau; ce qui correspond à une pression d'où l'emploi courant d'unité de pression pour mesurer le potentiel.

Il remarque aussi que la non-linéarité constatée dans la relation flux-potentiel tient pour une part au fait qu'il y a couplage entre flux soluté et flux d'eau. C'est du moins une des explications possibles.

En réponse à $M$. VERNET, $M$. BERGER indique que son schéma général de la circulation dans la plante n'est pas uniquement hypothétique mais découle des résultats de mesure qui ont été publiés. Il précise cependant n'avoir pas eu l'occasion de les comparer avec les données récentes concernant la circulation dans le phloème.

A la demande du Président, M. BERGER expose brièvement les techniques utilisées pour évaluer les différents potentiels de l'eau :

Une première technique consiste à couper une feuille, dont le limbe est placé dans une chambre étanche et le pétiole à l'extérieur. Le limbe est alors soumis à une montée régulière de pression de gaz, en général de l'azote. La valeur de la pression observée lorsque la sève brute apparaît au niveau de la section est considérée comme étant égale à la tension existant dans la colonne de sève avant la section de l'organe. En toute rigueur le potentiel hydrique de la sève est égal à la somme de cette tension et du potentiel osmotique de la sève. Ce dernier terme est en général négligé et l'on admet l'égalité entre potentiel et tension.

Une đeuxième méthode de mesure est basée sur l'équilibre entre le potentiel hydrique du substrat placé dans une chambre close et l'atmosphère de la chambre. Lorsque, condition isotherme, l'équilibre est atteint, un système psychrométrique à thermocouple permet de mesurer le déficit de saturation de l'air et calculer ensuite le potentiel hydrique du substrat. Dans ce cas, on mesure quelque chose de très global. S'il y a des différences entre les parties intracellulaires et extracellulaires, étant donné que l'équilibre de tension de vapeur demande une heure au minimum, elles disparaissent et on obtient une valeur moyenne.

Pour mesurer le potentiel osmotique, quand on broie une feuille, on mélange les deux compartiments. Le compartiment intracellulaire ayant vraisemblablement un volume liquide plus grand que le compartiment extracellulaire, la moyenne obtenue se rapproche beaucoup plus du potentiel osmotique du milieu intracellulaire que de celui extracellulaire. Il n'y a aucun moyen de mesure directe actuellement.

Quant au potentiel de turgescence dans les cellules il est mesuré en faisant la différence entre une mesure de potentiel global et une mesure de potentiel osmotique. Ces deux mesures étant entachées d'erreur la valeur obtenue n'est pas très bonne. 